\section{Health-Related Quality of Life in Patients With Sarcoma: Enhancing Personalized Medicine}

To THE EdITOR: We read with great interest the Special Series entitled "Novel Therapeutic and Diagnostic Advances in Bone and Soft Tissue Sarcomas." These articles provide comprehensive insight into current knowledge and evolving research in this heterogeneous group of tumors. Although relevant diagnostic and therapeutic aspects of patient care are considered, there is almost no reference to the patient perspective on these advances. Most authors acknowledge the need for individualized care, with respect to clinical, genetic, and molecular factors; however, only Gounder et $\mathrm{al}^{1}$ refer to health-related quality of life (HRQOL) measures as potentially meaningful clinical end points in locally advanced connective tissue tumors.

Historically, evaluation of oncologic treatments has focused on objective outcomes such as radiologic response, progressionfree and overall survival, and health care provider perspectives on treatment-related toxicities. More recently, increasing attention has been given to patient-reported outcomes (PROs), defined as "any report of the status of a patient's health condition that comes directly from the patient, without interpretation of the patient's response by a clinician or anyone else," ${ }^{2}(\mathrm{p} 2)$ to evaluate treatment efficacy. PROs include a range of outcomes such as symptoms, functioning, and HRQOL. HRQOL is the most widely used PRO and is a multidimensional concept that includes the patient's perception of the impact of the disease and its treatment on physical, psychological, and social functioning. ${ }^{3}$ Incorporating PROs into clinical practice can facilitate communication, improve symptom control and patient satisfaction, and reduce hospital admissions. ${ }^{4}$ A recent study in patients with metastatic solid tumors showed that routine PRO monitoring and immediate response to adverse events led to a 5-month survival benefit compared with standard care ${ }^{5}$ longer than that associated with most new drugs for metastatic cancers approved by the US Food and Drug Administration in $2016 .^{6}$

Data on HRQOL in patients with sarcoma are limited; however, many patients experience a substantial burden of physical and psychological symptoms, with an adverse impact on HRQOL. ${ }^{7}$ Integration of HRQOL with traditional measures of therapeutic response will provide a more comprehensive assessment of the efficacy and toxicity of novel therapies for patients with sarcoma. ${ }^{8}$ Symptomatic toxicities are frequently underreported by clinicians; therefore, PROs are a vital component of symptom detection, monitoring, and early intervention. ${ }^{9}$ Precise assessments of how patients feel and function offer important additional information in evaluating the risks and benefits of treatments. ${ }^{3}$ Patient experience is a key aspect of drug development, and survival alone is inadequate to determine net clinical benefit. ${ }^{10}$ Although some novel treatments have been approved for sarcomas over the last few decades, detailed data on short- and long-term adverse effects and HRQOL are scarce. ${ }^{7}$ The PALETTE (Pazopanib for Metastatic Soft Tissue Sarcoma) study of pazopanib versus placebo as second-line or greater treatment of advanced soft tissue sarcomas is one of the few sarcoma trials to report HRQOL as an exploratory end point. ${ }^{11}$ Pazopanib improved progression-free survival without relevant deterioration in HRQOL compared with placebo. This demonstrates that combining HRQOL with clinical data can show overall clinical treatment benefit.

One of the biggest challenges in sarcoma is how to assess HRQOL in this heterogeneous patient group. Previous studies have predominately used generic HRQOL instruments (European Organisation for Research and Treatment of Cancer Quality of Life Questionnaire Core 30, Short Form 36, Functional Assessment of Cancer Therapy-General); however, these tools do not efficiently capture the unique experiences of patients with sarcoma (eg, disease localization, treatment-specific symptoms) and thus lack content validity. Traditionally, inadequate content coverage has been addressed using a tumor-specific questionnaire that captures all disease-specific HRQOL issues, in conjunction with generic HRQOL measures. Given the heterogeneity of sarcomas, including patient age, histologic subtype, physiologic locations, disease stage, and rapidly changing treatment landscape, associated with variable mechanisms of action and toxicity profiles, it may be challenging to develop one sarcomaspecific questionnaire that meets the needs of clinical practice, academia, and industry. Standardized, so-called static, questionnaires consisting of a fixed set of items may not be relevant for every patient with sarcoma and may miss important patient-reported adverse events. Consequently, a more flexible approach is needed to assess the impact of treatments, provide optimal supportive care, and ultimately translate into meaningful outcomes for patients with sarcoma. ${ }^{12}$ One option is to combine standardized PRO questionnaires with validated items from item libraries (eg, Patient-Reported Outcomes Measurement Information System, European Organisation for Research and Treatment of Cancer) to ensure adequate assessment of specific treatments and their effects on common health problems. $^{12}$

In this era of personalized medicine, the principal focus has been on clinical and tumor characteristics, without addressing individual patient perspectives. If we really want to make a difference, truly provide personalized care, and conduct trials that are attractive to patients, we should routinely involve patients in trial design and integrate HRQOL assessments into clinical practice and research. ${ }^{13}$ This will enable provision of a more holistic approach in the overall management of patients.

\section{Eugenie Younger}

Royal Marsden Hospital, London, United Kingdom; and Radboud University Medical Center, Nijmegen, the Netherlands

\section{Roger Wilson}

Sarcoma Patients EuroNet, Riemerling, Germany

\section{Winette T.A. van der Graaf and Olga Husson}

Royal Marsden Hospital; Institute of Cancer Research, London, United Kingdom; and Radboud University Medical Center, Nijmegen, the Netherlands 


\section{AUTHORS' DISCLOSURES OF POTENTIAL CONFLICTS OF INTEREST}

Disclosures provided by the authors are available with this article at jco.org.

\section{REFERENCES}

1. Gounder MM, Thomas DM, Tap WD: Locally aggressive connective tissue tumors. J Clin Oncol 36:202-209, 2018

2. US Food and Drug Administration: Guidance for industry: Patient-reported outcome measures-Use in medical product development to support labeling claims. https://www.fda.gov/downloads/Drugs/GuidanceComplianceRegulatorylnformation/ Guidances/UCM193282.pdf

3. Kluetz PG, Slagle A, Papadopoulos EJ, et al: Focusing on core patientreported outcomes in cancer clinical trials: Symptomatic adverse events, physical function, and disease-related symptoms. Clin Cancer Res 22:1553-1558, 2016

4. Basch E, Spertus J, Dudley RA, et al: Methods for developing patientreported outcome-based performance measures (PRO-PMs). Value Health 18 493-504, 2015

5. Basch $E$, Deal AM, Dueck $A C$, et al: Overall survival results of a trial assessing patient-reported outcomes for symptom monitoring during routine cancer treatment. JAMA 318:197-198, 2017

6. US Food and Drug Administration: Hematology/oncology (cancer) approvals and safety notifications. https://www.fda.gov/Drugs/InformationOnDrugs/ ApprovedDrugs/ucm279174.htm
7. Chan A, Lim E, Ng T, et al: Symptom burden and medication use in adult sarcoma patients. Support Care Cancer 23:1709-1717, 2015

8. Reeve BB, Mitchell SA, Dueck AC, et al: Recommended patient-reported core set of symptoms to measure in adult cancer treatment trials. J Natl Cancer Inst 106:dju129, 2014

9. Di Maio M, Basch E, Bryce J, et al: Patient-reported outcomes in the evaluation of toxicity of anticancer treatments. Nat Rev Clin Oncol 13:319-325, 2016

10. Basch E, Dueck AC: Patient-reported outcome measurement in drug discovery: A tool to improve accuracy and completeness of efficacy and safety data. Expert Opin Drug Discov 11:753-758, 2016

11. Coens C, van der Graaf WT, Blay JY, et al: Health-related quality-of-life results from PALETTE: A randomized, double-blind, phase 3 trial of pazopanib versus placebo in patients with soft tissue sarcoma whose disease has progressed during or after prior chemotherapy: A European Organization for Research and Treatment of Cancer Soft Tissue and Bone Sarcoma Group global network study (EORTC 62072). Cancer 121:2933-2941, 2015

12. Groenvold M, Aaronson NK, Darlington AE, et al: Focusing on core patientreported outcomes in cancer clinical trials: Letter. Clin Cancer Res 22:5617, 2016

13. Wilson R. Patient led PROMS must take centre stage in cancer research. Res Involv Engagem 4:7, 2018

DOI: https://doi.org/10.1200/JC0.2018.77.9819; published at jco.org on April 18, 2018. 
AUTHORS' DISCLOSURES OF POTENTIAL CONFLICTS OF INTEREST

Health-Related Quality of Life in Patients With Sarcoma: Enhancing Personalized Medicine

The following represents disclosure information provided by authors of this manuscript. All relationships are considered compensated. Relationships are self-held unless noted. I = Immediate Family Member, Inst = My Institution. Relationships may not relate to the subject matter of this manuscript. For more information about ASCO's conflict of interest policy, please refer to www.asco.org/rwc or ascopubs.org/jco/site/ifc.

\section{Eugenie Younger}

No relationship to disclose

Roger Wilson

Honoraria: PharmaMar
Winette T.A. van der Graaf

Research Funding: GlaxoSmithKline (Inst), Novartis (Inst)

\section{Olga Husson}

No relationship to disclose 